ECCOMAS

Proceedia
COMPDYN 2019

$7^{\text {th }}$ ECCOMAS Thematic Conference on Computational Methods in Structural Dynamics and Earthquake Engineering M. Papadrakakis, M. Fragiadakis (eds.) Crete, Greece, 24-26 June 2019

\title{
COMPARISON BETWEEN MODELS FOR THE EVALUATION OF THE SEISMIC RESPONSE OF OFFSHORE WIND TURBINES ON DEEP FOUNDATIONS
}

\author{
Guillermo M. Álamo*, Jacob D.R. Bordón, Luis A. Padrón, Juan J. Aznárez and \\ Orlando Maeso
}

Instituto Universitario de Sistemas Inteligentes y Aplicaciones Numéricas en Ingeniería (SIANI) Universidad de Las Palmas de Gran Canaria

Edificio Central del Parque Científico y Tecnológico del Campus Universitario de Tafira 35017 Las Palmas de Gran Canaria, Spain

\{guillermo.alamo, jacobdavid.rodriguezbordon,luis.padron,juanjose.aznarez,orlando.maeso\}@ulpgc.es

\begin{abstract}
In this work, a comparative study between several numerical methodologies for the seismic analysis of offshore wind turbines on deep foundations (monobuckets or monopiles) is presented. Three formulations are compared within the scope of linear elasticity. First, a coupled Boundary Element - Finite Element (BEM-FEM) model is considered for obtaining the reference results. This methodology makes use of boundary elements to discretize the soil, while modelling the actual geometry of the hollow pile through shell finite elements. The second model corresponds to a formulation based on the integral expression of the reciprocity theorem in elastodynamics and the use of specific Green's functions for the layered half space for the modeling of soil, and the treatment of the pile as unidimensional beam elements. Finally, a Beam-on-Dynamic-Winkler-Foundation (BDWF) model is also considered as a commonly used simple tool for the analysis of deep foundations. The results are presented in terms of variables of interest for the design of offshore wind turbines. From the analyses, the applicability range of these methodologies can be established depending on the properties of the turbine-foundationsoil system.
\end{abstract}

Keywords: offshore wind turbines, bucket foundations, pile foundations, soil-structure interaction, seismic analysis 


\section{INTRODUCTION}

Pile and bucket (also known as suction buckets, caissons, piles or anchors depending on the context) foundations are being used as foundations of fixed Offshore Wind Turbines (OWT) [1]. These solutions configured as a single foundation element or multiple elements are considered at locations with shallow and intermediate water depths (up to 50 meters). Although both are topologically similar, i.e. steel tubular structures, they differ from each other in the diameter $D$, wall thickness to diameter ratio $t / D$, length to diameter ratio $L / D$ and hence also in the installation method. Typical monopiles for OWT are installed by pile driving using huge hydraulic hammers, and they have diameters around 3 to 6 meters (although XL monopiles with diameters up to 9 meters are also present in the industry), wall thickness to diameter ratios $t / D \approx 0.01$ $(1 \%)$ and length to diameter ratios $L / D \geq 5$. Typical buckets are installed by suction, and they have diameters around 5 to 15 meters, wall thickness to diameter ratios $t / D \approx 0.001(1 \%)$, and length to diameter ratios $L / D \leq 6[2,3]$ ( $L / D \approx 1$ for sands, $L / D \approx 3$ for stiff clays).

The use of large diameter and relatively short monopiles and buckets with such a thin-walled hollow cross-section is challenging from the modelling of the dynamic response point of view. In recent years, there has been a considerable effort for studying the influence of different factors (e.g. soil stratigraphy [4] and contact conditions [5]) and the characterisation of the dynamic response. To this end, rigorous continuum models based on the Boundary Element Method (BEM) or the Finite Element Method (FEM) have great generality and can be used, but they are time consuming at the pre-processing stage and computationally expensive at the solving stage. This forces the analyst to solve a limited number of cases, or to use (and sometimes misuse) other simpler models. For this reason, many models with different degrees of simplifying assumptions have been developed over the last years, see e.g. [6, 7, 8]. Particularly attractive are one-dimensional models, which are typically cheap to run. However, seismic inputs may contain energy in the range of frequencies with wavelengths equal or smaller than diameters, and thus it is not clear to what extent they remain valid for monopiles and buckets lately encountered.

The aim of this paper is to perform a comparative study between three foundation models (BEM-FEM model with soil-shell interaction [7], integral model with soil-beam interaction [8] and a Beam-on-Dynamic-Winkler-Foundation (BDWF) model [9]) for the evaluation of the seismic response of OWTs founded on deep (monopile and bucket) foundations.

The rest of the paper is organized as follows. Section 2 describes the problem at hand, while Section 3 describes the methodologies considered for its analysis. Section 4 contains a presentation and discussion of the obtained results. Finally, section 5 presents the conclusions.

\section{PROBLEM DEFINITION}

For the sake of conciseness, the present study is limited to the comparison between results from the previously mentioned three models for a fixed configuration where only foundation $L / D$ ratio is varied. Nonetheless, the fixed parameters are set to typical values which are representative of current OWTs.

The turbine-monopile-foundation system is defined based on the properties of the reference NREL-5MW OWT [10], see Fig. 1. The tower presents a hollow section with variable diameter that goes from $6 \mathrm{~m}$ at its base to $3.87 \mathrm{~m}$ at hub height. A constant thickness to diameter ratio of $0.45 \%$ is assumed. The tower length is $70 \mathrm{~m}$. The supporting structure corresponds to a monopile with a length of $20 \mathrm{~m}, 6 \mathrm{~m}$ diameter and a thickness ratio of $1 \%$. No transition piece is considered in the analyses. The foundation element (pile/bucket) presents the same diameter 

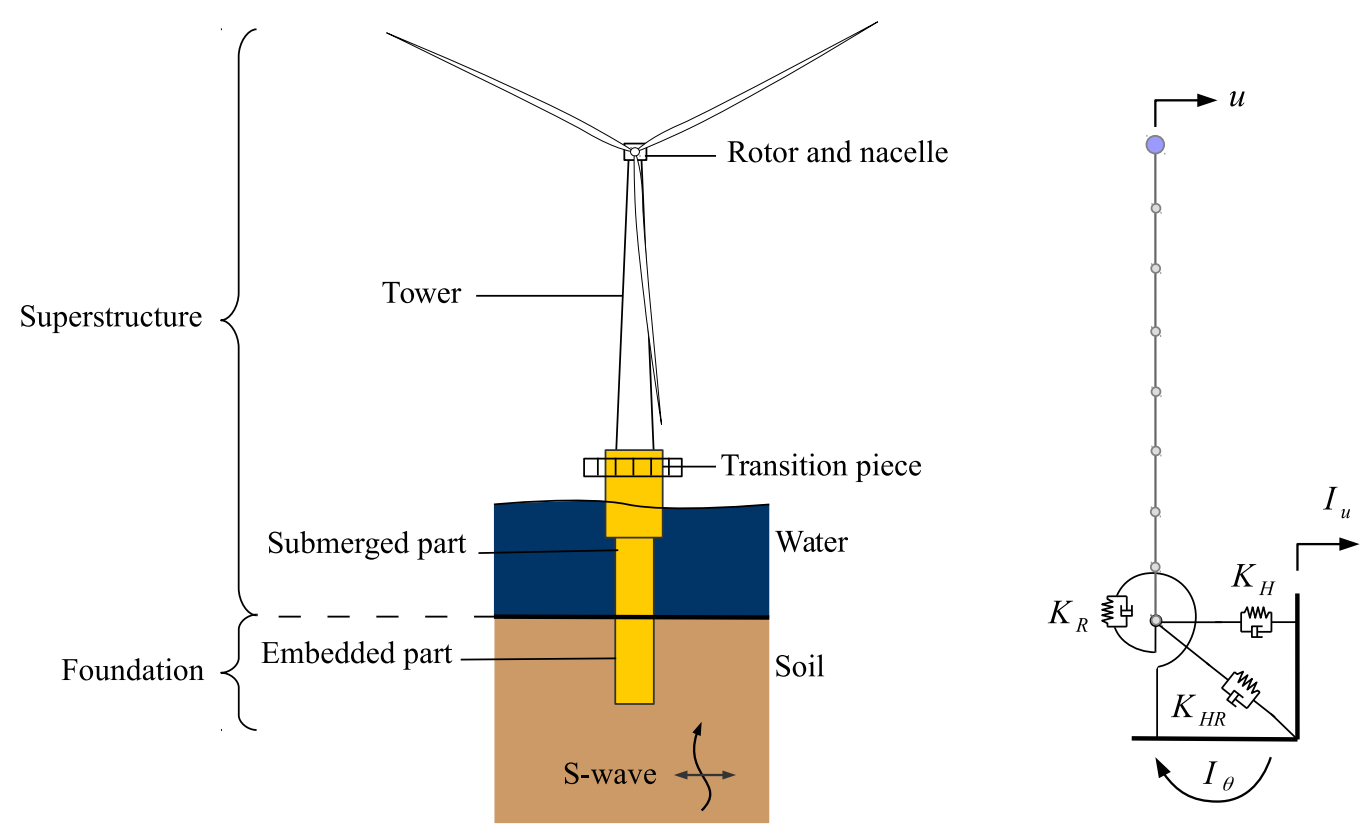

Figure 1: Problem layout and substructuring model

$D$ and thickness as the monopile, while various embedment lengths $L$ are assumed in order to cover the typical range of aspect ratios ( $L / D$ from 2 to 10$)$, see Fig. 2a.

Steel material properties are considered for the tower, supporting monopile and foundation element: Young's modulus $210 \mathrm{GPa}$, density $7850 \mathrm{~kg} / \mathrm{m}^{3}$ and Poisson's ratio 0.25 . For the superstructure an hysteretic damping coefficient $2.5 \%$ is considered.

The soil properties are selected in order to reproduce a saturated media through elastic equivalent properties: shear wave velocity $200 \mathrm{~m} / \mathrm{s}$, density $=2000 \mathrm{~kg} / \mathrm{m}^{3}$, Poisson's ratio 0.49 and hysteretic damping coefficient $5 \%$.

The considered seismic excitation is a vertically incident shear wave. The free-field wave motion is denoted as $u_{f f}$.

\section{METHODOLOGY}

\subsection{Substructuring model}

The seismic response of the OWT-foundation system is computed through a 2-D substructuring model, see Fig. 1. The superstructure, i.e. tower and supporting pile, is modelled with Finite Elements. Two-noded Bernoulli beam elements with constant-section are considered. A high-enough number of them to adequately represent the dynamic behaviour of the structure and the stiffness of the conical tower is used. The mass of the rotor and nacelle is included as a point mass added at the highest node of the tower.

The soil-foundation interaction is included through the impedance functions and kinematic interaction factors computed by each foundation model, see Fig. 2. The firsts represent the relation between the forces (moments) and displacements (rotations) produced atop the foundation element. These impedance functions are frequency-dependent complex values whose real and imaginary components represent the stiffness and damping of the foundation, respectively. Because only the lateral behaviour of the system is studied, just the horizontal $K_{H}$, rocking $K_{R}$ and coupled horizontal-rocking $K_{H R}$ impedance functions are considered. On the other hand, the kinematic interaction factors represent the filtering effects of the foundation and are computed 
as the relation of the displacement (or rotation) at the head of the pile/bucket and the free-field motion. In this work, the translational and rotational kinematic interaction factors are denoted as $I_{u}$ and $I_{\theta}$, respectively.

Finally, for comparison purposes, it would be also interesting to compute the response of the system neglecting the SSI effect, i.e. under the rigid base assumption. In order to do so, the free field motion is directly applied at the mud-line node of the superstructure, while restricting its rotation.

\subsection{Foundation models}

\subsubsection{BEM-FEM model with soil-shell interaction}

Reference results are obtained from a rigorous BEM-FEM model [7] which considers the interaction between the pile and the soil as the interaction of the pile shell and the surrounding (interior and exterior) soil, see Fig. 2b. The only assumption is related to the shell interaction, which is reduced to the mid-surface of the shell. Taking into account that $t / D$ ratios are typically well below 5\%, it is virtually a continuum model. A concise description of this model is outlined below, for more details see [7].

The soil region $\Omega_{\mathrm{s}}$ is treated using the BEM. The BEM is based on the collocation of Boundary Integral Equations (BIE) relating displacements $u_{k}$ and tractions $t_{k}$ throughout the boundary of the region. The boundary of $\Omega_{\mathrm{s}}$ is $\Gamma=\partial \Omega_{\mathrm{s}}$, which is composed in two parts: free-surface boundary $\Gamma_{\mathrm{fs}}$, and shell mid-surface $\Gamma_{\mathrm{sm}}$ considered as a crack-like boundary $\left(\Gamma_{\mathrm{sm}}=\Gamma_{\mathrm{sm}}^{+}+\Gamma_{\mathrm{sm}}^{-}\right)$. The Singular BIE is used for collocating along the free-surface:

$$
c_{l k}^{\mathrm{i}} u_{k}^{\mathrm{i}}+\int_{\Gamma} t_{l k}^{*} u_{k} \mathrm{~d} \Gamma=\int_{\Gamma} u_{l k}^{*} t_{k} \mathrm{~d} \Gamma+\int_{\Omega_{\mathrm{s}}} u_{l k}^{*} b_{k} \mathrm{~d} \Omega_{\mathrm{s}}
$$

where $l, k=1,2,3$ and the summation convention is implied. Furthermore, $c_{l k}^{\mathrm{i}}$ is the free-term at the collocation point, $u_{k}^{\mathrm{i}}$ is the displacement at the collocation point, $u_{l k}^{*}$ and $t_{l k}^{*}$ are the fullspace elastodynamic fundamental solutions in terms of displacements and tractions respectively (see e.g. [11]), and $b_{k}$ are the body forces acting over the domain $\Omega_{\mathrm{s}}$ assumed to be zero for the present formulation. The Dual (Singular and Hypersingular) BIEs are used for collocating along the shell mid-surface:

$$
\begin{aligned}
& \frac{1}{2}\left(u_{l}^{\mathrm{i}+}+u_{l}^{\mathrm{i}-}\right)+\int_{\Gamma} t_{l k}^{*} u_{k} \mathrm{~d} \Gamma=\int_{\Gamma} u_{l k}^{*} t_{k} \mathrm{~d} \Gamma \\
& \frac{1}{2}\left(t_{l}^{\mathrm{i}+}-t_{l}^{\mathrm{i}-}\right)+\int_{\Gamma} s_{l k}^{*} u_{k} \mathrm{~d} \Gamma=\int_{\Gamma} d_{l k}^{*} t_{k} \mathrm{~d} \Gamma
\end{aligned}
$$

where $u_{k}^{\mathrm{i}+}, t_{k}^{\mathrm{i}+}$ and $u_{k}^{\mathrm{i}-}, t_{k}^{\mathrm{i}-}$ are displacements and tractions at the collocation point on respectively the positive and negative crack faces, and $d_{l k}^{*}$ and $s_{l k}^{*}$ are obtained from the differentiation of $u_{l k}^{*}$ and $t_{l k}^{*}$ (see e.g. [11]). In Eqs. (2-3), it is assumed that the collocation point $\mathbf{x}^{\mathrm{i}}$ is located at a smooth boundary point $\left(\Gamma_{\mathrm{sm}}\left(\mathbf{x}^{\mathrm{i}}\right) \in \mathscr{C}^{1}\right)$, which leads to the $1 / 2$ factor. This assumption is related to the use of Multiple Collocation Approach (MCA) [12] when collocating at a crack boundary point. Lagrange quadratic triangular (6 nodes) and quadrilateral ( 9 nodes) boundary elements are used for the discretisation. The pile shell region $\Omega_{\mathrm{ps}}$ is discretised using the FEM. Shell finite elements based on the degeneration from the solid are considered, and the lockingphenomena is overcome by using the Mixed Interpolation of Tensorial Components (MITC). In 


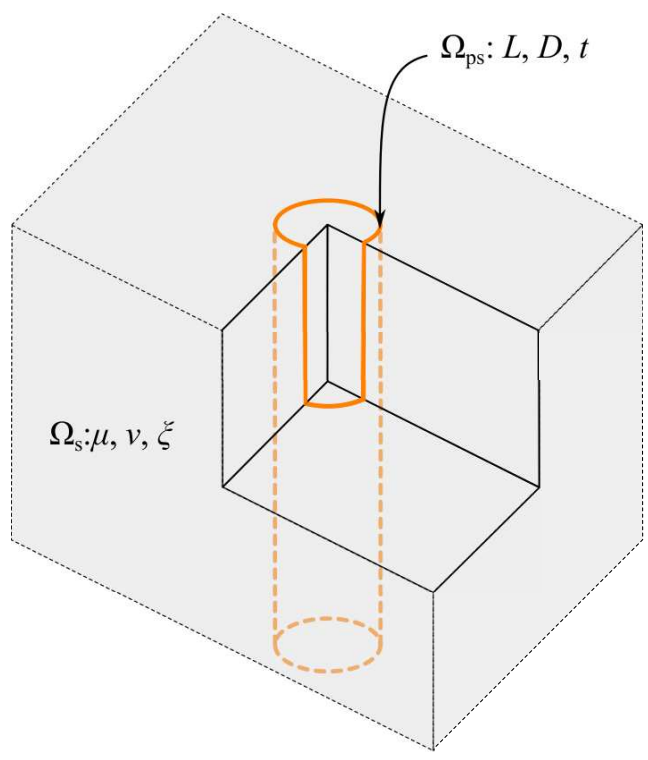

(a)

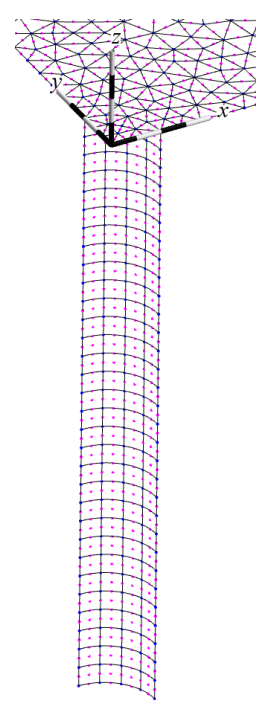

(b)

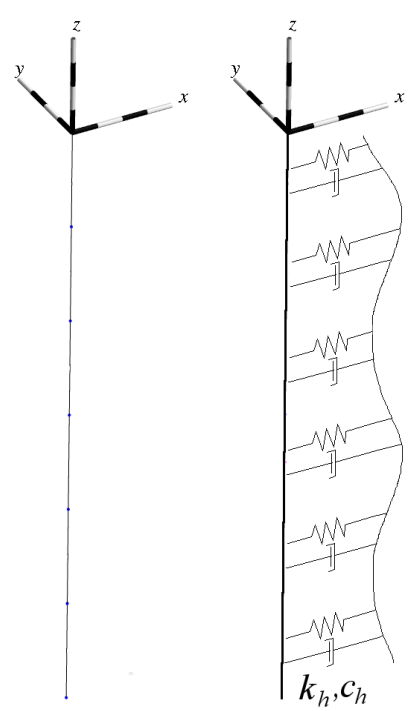

(c)

(d)

Figure 2: Foundation configuration and its modelling: (a) steel hollow pile in homogeneous half-space, (b) DBEM-FEM model (soil-shell interaction), (c) Integral model (soil-beam interaction) and (d) Winkler model (soil-beam interaction)

particular, the MITC9 shell finite element [13] is used in this work. The equilibrium equation for a given shell finite element $l$ can be written as:

$$
\tilde{\mathbf{K}}^{(l)} \mathbf{a}^{(l)}-\mathbf{Q}^{(l)} \mathbf{t}^{(l)}=\mathbf{f}^{(l)}
$$

where $\tilde{\mathbf{K}}^{(l)}=\mathbf{K}^{(l)}-\omega^{2} \mathbf{M}^{(l)}$ is the resulting time harmonic stiffness matrix, $\mathbf{a}^{(l)}$ is the vector of nodal displacements and rotations, $\mathbf{Q}^{(l)}$ is the matrix converting distributed mid-surface load $\mathbf{t}^{(l)}$ into nodal loads, and $\mathbf{f}^{(l)}$ is the vector of equilibrating nodal forces. Finally, coupling is performed by imposing compatibility and equilibrium between shell finite element and cracklike boundary:

$$
\begin{array}{r}
u_{k}^{+}=u_{k}^{-}=u_{k}^{l} \\
t_{k}^{+}+t_{k}^{-}+t_{k}^{l}=0
\end{array}
$$

where $u_{k}^{l}$ denotes the shell displacements and $t_{k}^{l}$ the distributed mid-surface shell load.

The seismic excitation is included in the formulation following the classical decomposition of the total field into the superposition of the incident field (produced by the impinging waves) and the scattered field (produced by the presence of the foundation) [11].

\subsubsection{Integral model with soil-beam interaction}

With the intention of reducing the number of degrees of freedom needed to solve the soilfoundation problem, a second numerical model [8] is considered. The main simplification of this model with respect to the previous BEM-FEM one is the treatment of the foundation (pile/bucket) as a beam element. By doing so, the discretisation of the soil-foundation interface is avoided and the soil-foundation interaction is reduced to distributed loads $q_{k}$ acting over the load line $\Gamma_{1}$ that represents the foundation element. On the other hand, the integral model 
makes use of specific Green's functions for the layered half space [14] as fundamental solutions instead of the aforementioned ones corresponding to the full-space. This substitution avoids the discretisation of the free-surface, as the particular Green's functions already satisfy the zero traction boundary condition. Fig. 2c shows how simple is the required discretisation for this model. Thus, the Singular BIE that is used for collocating along the foundation-load line can be reduced to:

$$
u_{l}^{\mathrm{i}}=\int_{\Gamma_{1}} \tilde{u}_{l k}^{*} q_{k} \mathrm{~d} \Gamma_{1}
$$

where $\tilde{u}_{l k}^{*}$ is the displacement fundamental solution for the layered half space [14]. A special non-nodal collocation strategy is required for numerically evaluating the integral of the left-hand side (see [8] for more details). The load line $\Gamma_{1}$ is discretised using the two-noded finite elements. Cubic and quadratic shape functions that satisfy the Timoshenko's beam static equation [15] are used for the lateral behaviour, while linear shape functions are used to model the interaction tractions and axial displacements. The equilibrium equation for a given beam element $l$ can be written as:

$$
\tilde{\mathbf{K}}^{(l)} \mathbf{a}^{(l)}-\mathbf{Q}^{(l)} \mathbf{q}^{(l)}=\mathbf{f}^{(l)}
$$

being the terms of this equation the beam-counterparts of the ones presented in Eq. (4). Finally, the coupling between the foundation and soil is made by imposing compatibility and equilibrium conditions between the pile nodes and the corresponding points of the soil:

$$
\begin{array}{r}
u_{k}=u_{k}^{l} \\
q_{k}+q_{k}^{l}=0
\end{array}
$$

where $u_{k}^{l}$ denotes the beam displacements and $q_{k}^{l}$ the distributed interaction traction acting over the beam.

As in the BEM-FEM model, the seismic excitation is considered by the superposition of the incident and scattered fields.

\subsubsection{Beam-on-Dynamic-Winkler-Foundation model}

In order to compare the previous models against a well-established one, a classic Beam-onDynamic-Winkler-Foundation model (also known as Winkler model) is considered, see Fig. 2 d. It consists in solving the differential equation of the Euler-Bernoulli beam using a distributed load representing the soil reaction. This is introduced as distributed springs and dashpots taken from $[9,16]$. Such Winkler model is a very practical engineering approach to the problem, but requires the calibration of the distributed dynamic stiffnesses based on fitting of numerical results (as in $[9,16])$ or some analytical solution of simpler problems (e.g. [17]).

\section{RESULTS AND DISCUSSION}

First, results corresponding to the rigid base assumption are presented. Table 1 shows the natural frequencies and the amplification ratios at hub height for the first 4 modes of the turbinesupporting structure system. The complete harmonic response of the system is depicted by the grey solid line in Fig. 3.

Fig. 3 also shows the harmonic response obtained by considering the different models of the foundation: BEM-FEM (black line), integral (orange line) and Winkler (blue dashed line). 


\begin{tabular}{ccc}
\hline mode & $f_{n}^{\text {rigid }}(\mathrm{Hz})$ & $u_{\max }^{\text {rigid }} / u_{f f}$ \\
\hline 1 & 0.369 & 22.3 \\
2 & 3.33 & 4.26 \\
3 & 7.14 & 2.75 \\
4 & 14.5 & 1.18 \\
\hline
\end{tabular}

Table 1: Natural frequencies and harmonic hub displacement under the rigid base assumption.
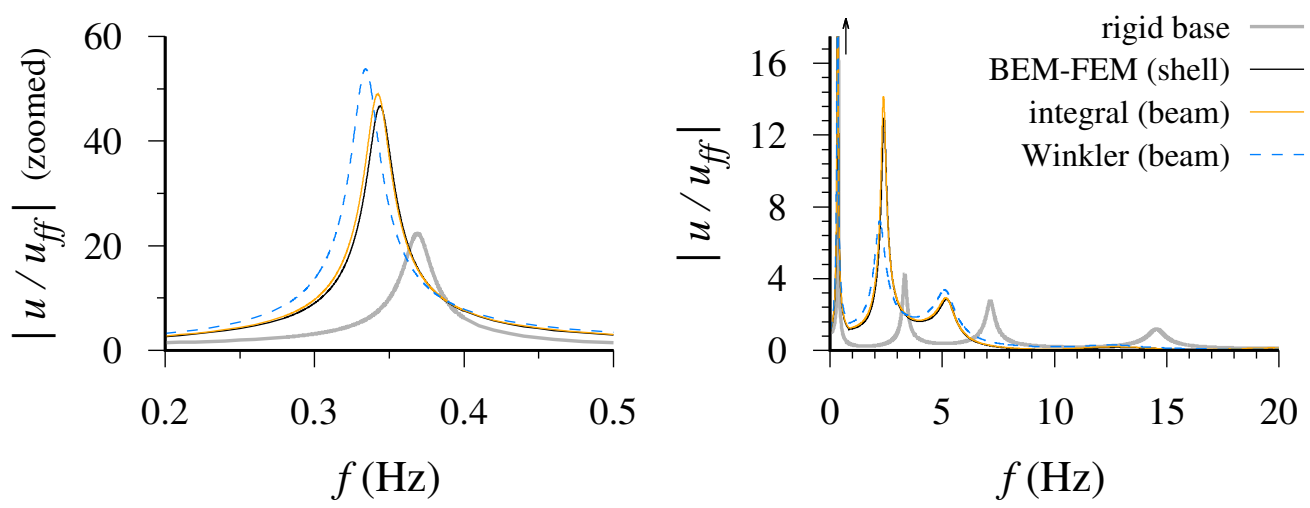

Figure 3: Harmonic seismic response of the OWT. Comparison between the three foundation models and the rigid base assumption. $L / D=6$.

For all of them an aspect ratio $L / D=6$ has been considered for the foundation element. The results show a strong influence of the soil-structure interaction (SSI) effects, not only in the shifts towards lower natural frequencies, but also in the increments of the amplification of the soil free-field motion. Regarding the use of the different models, the BEM-FEM and integral formulations lead to similar responses, while the results of the Winkler model slightly diverge.

The quantification of the importance of the soil-structure interaction effects and the modelling of the foundation is summarized in Fig. 4. This figure displays the relation between the soil motion amplification ratio considering or not the SSI effects, as well as the relation between the natural frequencies obtained for the flexible and rigid base. The results of the different models are presented following the same colors as before and different aspect ratios are considered along the different columns. Only the first three modes are studied as they are found to be the relevant ones when the SSI effects are taken into account (see Fig. 3). As commented before, the results obtained by the BEM-FEM and integral model present less differences than the ones obtained by the simpler Winkler approach. The second mode is found to be the most sensitive to the foundation model, especially in terms of the amplification ratio. Regarding the effects of the foundation aspect ratio, as expected, larger differences between the shell and beam models are found for the smallest $L / D$.

In order to understand the differences between the three models, the impedance functions and kinematic interaction factors obtained by each of them are presented in Figs. 5 and 6, respectively. Only the frequency range of interest is plotted.

The comparison between the BEM-FEM and integral models shows agreeing results both in terms of impedance functions and kinematic interaction factors. Only appreciable differences are found in the kinematic interaction factors for the configuration with $L / D=2$ due to the 

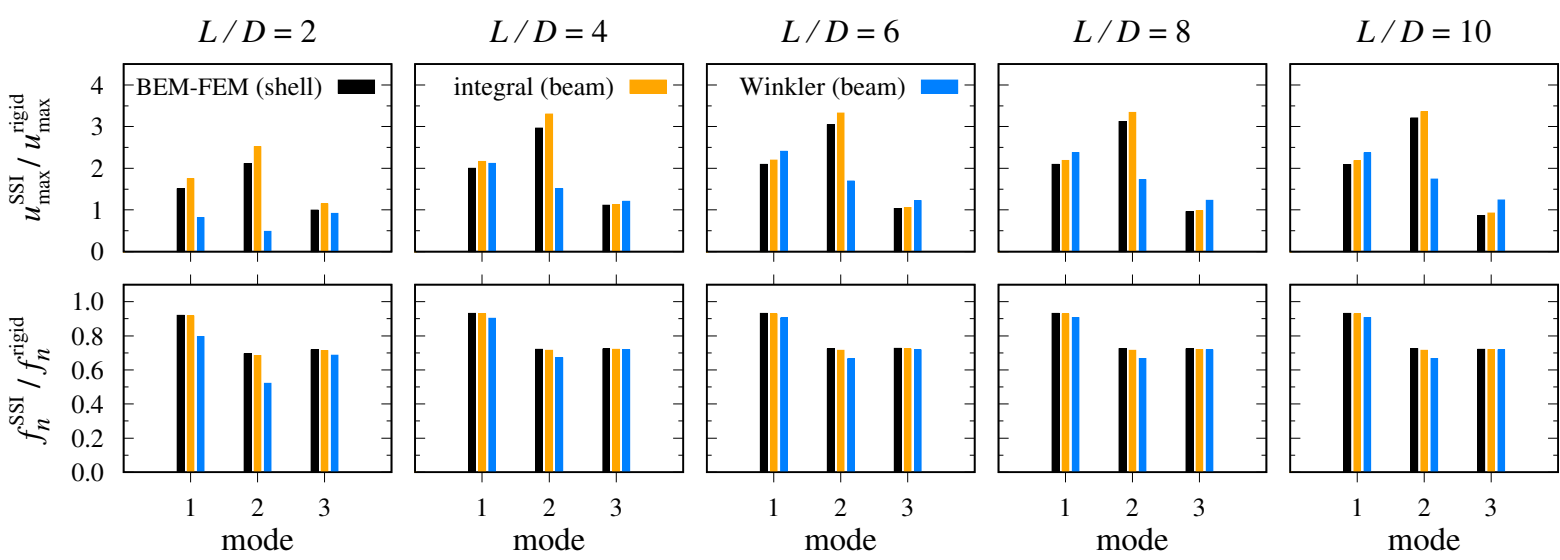

Figure 4: Influence of SSI effects on the estimation of the maximum seismic response and natural frequencies of the OWT. Comparison between the three foundation models.

inability of the beam model to reproduce the behaviour of the short foundation. For this configuration, the larger input motion obtained by the integral model explains the larger response presented in Fig. 4.

On the other hand, the Winkler model can not closely reproduce the reference results of the BEM-FEM formulation. Large differences are found in the impedance terms involving rotations and also in the imaginary component (i.e., foundation damping) of all impedance terms. The discrepancies of the Winkler model become more evident for the kinematic interaction factors, presenting larger input displacements and rotations than the reference foundation model as the frequency increase. The combination of these two opposite effects makes that, depending on the mode and configuration, the Winkler model leads to larger (when the increase in input motion is more important) or smaller (when the increase in the foundation damping is more important) amplification ratios with respect to the ones of the BEM-FEM model.

\section{CONCLUSIONS}

The present contribution presents a comparison between three models of different modelling approximation levels for pile/bucket foundations under seismic excitation. The reference BEMFEM model considers the complete soil-shell interaction [7], while the other models (integral model and Winkler model) consider a soil-beam interaction. The integral model [8] rigorously incorporates the soil using a Green's function, while the Winkler model uses a tuned distribution of springs and dashpots from $[9,16]$.

In general terms, it is observed that the integral model is superior to the Winkler model, although in some cases the discrepancies are small enough for rough and quick analyses. Surprisingly, it is also observed that soil-beam models roughly reproduces the results for small $L / D$.

\section{ACKNOWLEDGEMENTS}

This work was supported by Subdirección General de Proyectos de Investigación del Ministerio de Economía, Industria y Competitividad (MINECO) and Agencia Estatal de Investigación (AEI) of Spain and FEDER through research project BIA2017-88770-R and by Universidad de Las Palmas de Gran Canaria and Consejería de Economía, Industria, Comercio y Conomiciento del Gobierno de Canarias through research project CEI2018-9. 

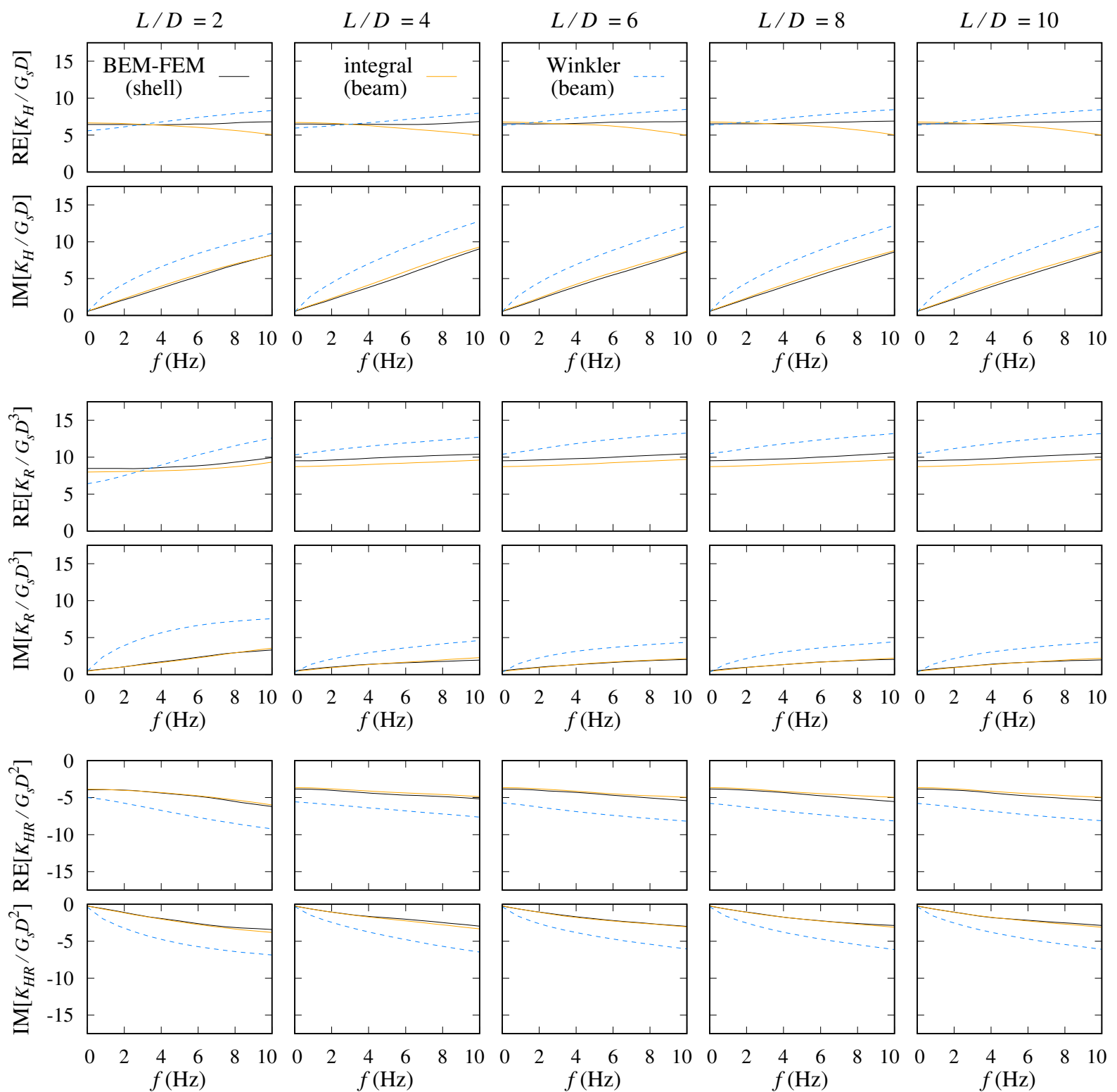

Figure 5: Horizontal, rocking and coupled horizontal-rocking impedance functions. Comparison between the three foundation models. 

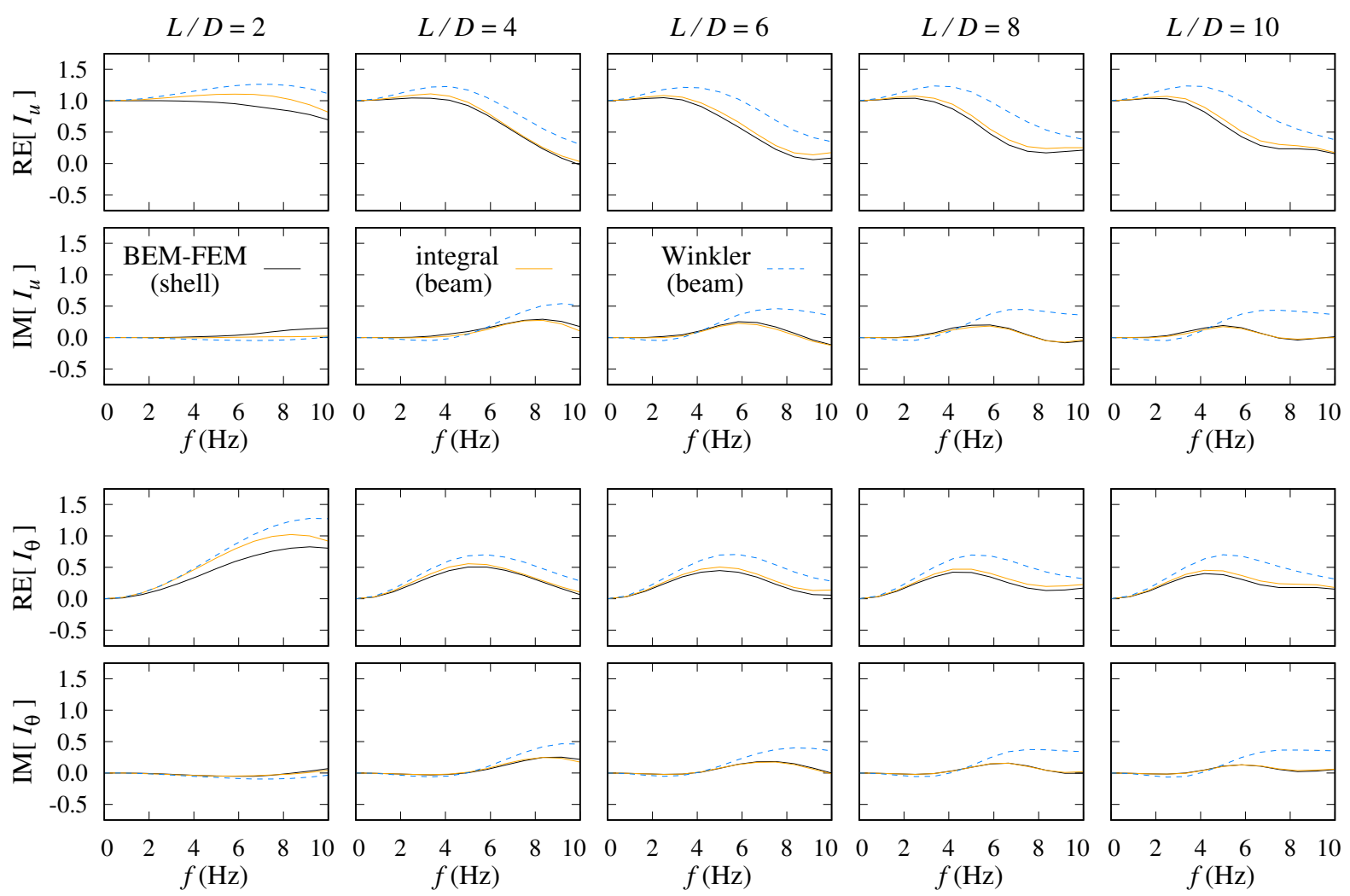

Figure 6: Translational and rotational kinematic interaction factors. Comparison between the three foundation models.

\section{REFERENCES}

[1] K.-Y. Oh, W. Nam, M. S. Ryu, J.-Y. Kim, B. Epureanu. A review of foundations of offshore wind energy convertors: Current status and future perspectives. Renewable and Sustainable Energy Reviews, 88, 16-36, 2018.

[2] G.T. Houlsby and B.W. Byrne. Design procedures for installation of suction caissons in clay and other materials. Geotechnical Engineering, 158, 75-82, 2005.

[3] G.T. Houlsby and B.W. Byrne. Design procedures for installation of suction caissons in sand. Geotechnical Engineering, 158, 135-144, 2005.

[4] G.M. Álamo, J.J. Aznárez, L.A. Padrón, A.E. Martínez-Castro, F. Gallego and O. Maeso. Dynamic soil-structure interaction in offshore wind turbines on monopiles in layered seabed based on real data. Ocean Engineering, 156, 14-28, 2018.

[5] G.M. Álamo, J.D.R. Bordón, J.J. Aznárez and O. Maeso. Relevance of soil-pile tangential tractions for the estimation of kinematic seismic forces: Formulation and setting of a Winkler approach. Applied Mathematical Modelling, 59, 1-19, 2018.

[6] M. Shadlou and S. Bhattacharya. Dynamic stiffness of pile in a layered elastic continuum. Géotechnique, 64, 303-319, 2014.

[7] J.D.R. Bordón, J.J. Aznárez and O. Maeso. Dynamic model of open shell structures buried in poroelastic soils. Computational Mechanics, 60, 269-288, 2017. 
[8] G.M. Álamo, A.E. Martínez-Castro, L.A. Padrón, J.J. Aznárez, R. Gallego and O. Maeso. Efficient numerical model for the computation of impedance functions of inclined pile groups in layered soils. Engineering Structures, 126, 379-390, 2016.

[9] G. Gazetas and R. Dobry. Horizontal response of piles in layered soils. Journal of Geotechnical Engineering, 110(1), 20-40, 1984.

[10] J. Jonkman, S. Butterfield, W. Musial and G. Scott. Definition of a 5-MW reference wind turbine for offshore system development. Technical Report No. NREL/TP-500-38060, National Renewable Energy Laboratory, 2009.

[11] J. Domínguez. Boundary Elements in Dynamics. WIT Press, 1993.

[12] M.P. Ariza and J. Domínguez. General BE approach for three-dimensional dynamic fracture analysis. Engineering Analysis with Boundary Elements, 26, 639-651, 2002.

[13] M.L. Bucalem and K.J. Bathe. Higher-order MITC general shell elements. International Journal for Numerical Methods in Engineering, 36, 3729-3754, 1993.

[14] R.Y.S. Pak and B.B. Guzina. Three-dimensional Greens functions for a multilayered halfspace in displacement potentials. Journal of Engineering Mechanics, 128(4), 449-461, 2002.

[15] Z. Friedman and J.B. Kosmatka. An improved two-node Timoshenko beam finite element. Computers and Structures, 47(3), 473-481, 1993.

[16] R. Dobry, M.J. O’Rourke, J.M. Roesset and E. Vicente. Horizontal stiffness and damping of single piles. Journal of the Geotechnical Engineering Division, 108, 439-459, 1982.

[17] M. Novak , T. Nogami and F. Aboul-Ella. Dynamic soil reaction for plane-strain case, Journal of the Engineering Mechanics Division, 104, 953-959, 1978. 